\title{
Actuación preventiva de diagnóstico y control de movimientos en los edificios afectados por subsidencia en el barrio de la Estación de Sallent (Barcelona)
}

\author{
Preventative diagnosis and movement control in the buildings affected \\ by subsidence in La Estación neighborhood in Sallent (Barcelona) \\ C.Díaz $^{(*)}$, C. Cornadó ${ }^{(*)}$, P. Santamaría ${ }^{(*)}$, J. R. Rosell ${ }^{(*)}$, A. Navarro ${ }^{(*)}$
}

\begin{abstract}
RESUMEN
El presente artículo se refiere a la problemática de subsidencia surgida en los edificios de un barrio de la población de Sallent como consecuencia de los efectos de la excavación de las galerías subterráneas de una antigua explotación minera. Las necesarias medidas preventivas requeridas para hacer frente a dicha problemática propiciaron el conocimiento exhaustivo de las características técnico constructivas de los edificios, de sus daños y de la dinámica de sus afectaciones y condiciones de seguridad a lo largo del periodo de tiempo anterior a su definitivo desalojo. En el artículo se expone el proceso seguido para la toma de decisiones. Los datos obtenidos permiten, por una parte, conocer la relación entre los movimientos del terreno y los de los edificios, y por otra contrastar las formas reales de comportamiento de los edificios afectados frente a solicitaciones generadas por asentamientos diferenciales en sus firmes de cimentación.
\end{abstract}

Palabras clave: Patología de la edificación; subsidencia; asentamientos diferenciales; instrumentación de edificios.

\section{ABSTRACT}

The present paper deals with the subsidence problem arising in buildings of a neighborhood of Sallent (Barcelona, Spain) due to the excavation of an old mine tunnels. The necessary preventative measures required to deal with this problem prior to the final evacuation of the buildings led to the thorough understanding of their technical characteristics, their damage, and the dynamics of their cracks and their safety conditions. The article presents the methodology that was followed for decision making. The data obtained allow us to know on one hand, the relationship between the movements of the soil and the buildings, and on the other, to contrast the actual behavior of the buildings due to the stresses generated by the differential settlement in their foundations.

Keywords: Building pathology; subsidence; settlements; building instrumentation.

(*) Universitat Politècnica de Catalunya (UPC). Barcelona (España).

Persona de contacto/Corresponding author: cesar.diaz@upc.edu (C. Díaz)

Cómo citar este artículo/Citation: Díaz, C., Cornadó, C., Santamaría, P., Rosell, J. R., Navarro, A. (2015). Actuación preventiva de diagnóstico y control de movimientos en los edificios afectados por subsidencia en el barrio de la Estación de Sallent (Barcelona). Informes de la Construcción, 67(538): eo89, doi: http://dx.doi.org/10.3989/ic.13.168.

Licencia / License: Salvo indicación contraria, todos los contenidos de la edición electrónica de Informes de la Construcción se distribuyen bajo una licencia de uso y distribución Creative Commons Reconocimiento no Comercial 3.o. España (cc-by-nc). 


\section{INTRODUCCIÓN}

El barrio de la Estación de Sallent constituye uno de los ejemplos más característicos de afectación de una zona urbana por una problemática geológica generada por el movimiento de subsidencia del terreno en donde se ubica. Dicha subsidencia se manifiesta en superficie coincidiendo con la zona de excavación de las galerías subterráneas de una importante explotación minera iniciada en el año 1932, denominada Mina Enrique, destinada a la extracción de potasas. La Mina Enrique desciende hasta profundidades de unos 280 metros diseminada en numerosas galerías (1), ocupando una amplia zona que abarca el barrio de la Estación y que se extiende por el vecino barrio de La Rampiña, dentro del ámbito territorial de la denominada Cuenca Potásica Catalana.

A los pocos años de iniciarse los trabajos de excavación, algunos de los mineros empezaron a construir sus hogares en las afueras de Sallent, en los solares que cubrían la explotación minera propiedad de los concesionarios (Figura 1). En años sucesivos el barrio fue desarrollándose por dichos terrenos hasta alcanzar una superficie aproximada de 11,50 ha siguiendo un trazado de calles ortogonales con la inclusión de una plaza en disposición central. Hacia el año 2000, el barrio llegó a albergar unos 560 habitantes, distribuidos en 333 viviendas. La tipología edificatoria dominante es la casa entre medianeras de una a tres plantas de altura y uso familiar, si bien en el perímetro del barrio se construyeron también tres bloques de viviendas de 4 plantas y algunos grupos de viviendas unifamiliares en hilera. La explotación minera no se interrumpió hasta el año 1974 a causa de las abundantes avenidas de agua de imposible canalización que impedían la circulación interna y la ejecución de los trabajos en el interior de la mina.

\section{CARACTERÍSTICAS GEOLÓGICAS DEL TERRENO}

Los numerosos estudios geológicos y geotécnicos realizados (1) (2) (3) establecen con detalle y claridad que los edificios del barrio de la Estación se sitúan sobre materiales del Terciario continental, del Eoceno superior y del Oligoceno inferior, que engloban rocas sedimentarias de precipitación tipo calizas y margas de marcada estratificación, que forman el techo de la mina, y las evaporitas (sales explotadas en la mina). La parte superior de estos materiales es dónde se asientan la mayor parte de los edificios del barrio.

Sobre estos materiales se encuentra un suelo cuaternario, de 4 a 8 metros de profundidad, formado por tierra vegetal y suelos gravosos, propios de su ubicación en la cuenca fluvial del río Llobregat.

En el estrato rocoso se aprecia una marcada fracturación de las rocas de dirección vertical, relacionadas con las macroestructuras tectónicas que afectan a esta zona, como la falla del Yeso (que transcurre por el sur de los barrios de la Estación y La Rampinya), y la falla de Schwartz, perpendicular a la primera, de la cual se tiene constancia de su existencia en los trabajos de extracción minera y prospección geotécnica de la mina Enrique. Esta última falla, de componente casi vertical, juega un papel muy importante en la circulación hídrica subterránea, ya que por ella se infiltra el agua que circula por el torrente de Soldevila, lixiviando los sedimentos salinos, llegando incluso a formar una cavidad de unos 50 metros de altura que se halla en el extremo sud-este del barrio y a una profundidad de unos 145 metros (Figura 1). Esta cavidad - que se le reconoce con el nombre de «Gran Cavidad» - se fue engrandeciendo condi-
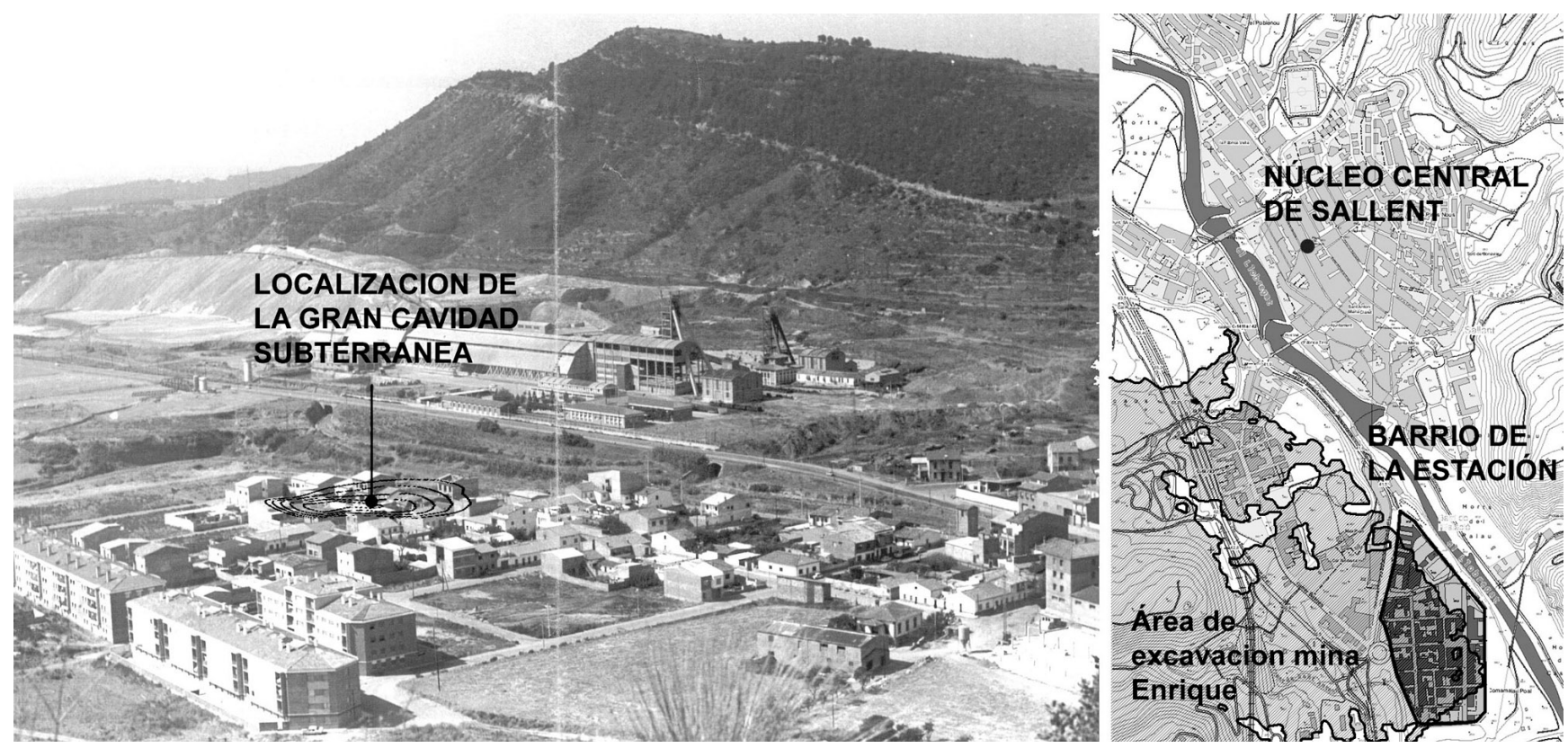

Figura 1. Vista general y plano de situación del barrio de la Estación hacia 1965, junto al río Llobregat, en el margen opuesto al núcleo urbano de Sallent.

\footnotetext{
Cabe citar entre dichos estudios los realizados por los geólogos Batlle y Mascareñas encargado por el Ayuntamiento de Sallent cuando se iniciaron de forma ostensible los daños en los edificios, el exhaustivo estudio de carácter institucional realizado por el Institut Cartográfic de Catalunya finalizado en 2002, con conclusiones fundamentadas sobre datos recopilados durante más de 3 años por las redes de control extensométrico y taquimétrico, así como el informe geológico, geotécnico y de riesgos geológicos finalizado en 2008, recopilatorio de toda la información obtenida y actualizada, realizado por los geólogos R. Mata y M. Puiguriguer, coordinados por el catedrático J. M. Mata-Perelló, en convenio con el Ayuntamiento de la Vila de Sallent para realizar el Plan de ordenación urbanística municipal.
} 
cionada por factores antrópicos como la abertura de galerías mineras y cambios en los sistemas de drenaje subterráneos.

\section{LA PROBLEMÁTICA DE LA SUBSIDENCIA}

La excavación de la mina Enrique se prolongó desde 1932 hasta 1974, año en que fue suspendida su explotación por la imposibilidad de encauzar las penetraciones de agua que impedían los trabajos de extracción y acarreo del mineral. A lo largo de este periodo, la disolución de las margas inducida por la presencia de agua, unido a los procesos geológicos típicos de descompresión de los terrenos situados por encima de las galerías excavadas, originó desde sus orígenes la activación de procesos de subsidencia detectables en superficie. Procesos que no se interrumpieron en períodos posteriores a las operaciones de relleno de las galerías con salmuera saturada en cloruro sódico llevadas a cabo durante los años 1976-77, una vez abandonadas las operaciones de extracción, confiando en la reacción de este compuesto con la carnalita presente en el terreno para conseguir su estabilización.

Desde los inicios de la explotación, las empresas mineras realizaron campañas de nivelación para disponer de información sobre los efectos de la subsidencia en superficie. Los datos disponibles referentes al barrio de la Estación indican una velocidad media de subsidencia comprendida entre 1 y $2,5 \mathrm{~cm} /$ año considerando el periodo 1948-1988, e indican también una tendencia a alcanzar valores inferiores a $2 \mathrm{~cm} / \mathrm{año} \mathrm{en} \mathrm{la}$ década 1965-75. De todas formas, dicha tendencia, según se constató en las nivelaciones realizadas posteriormente, se invirtió en algunas zonas del barrio en los años posteriores al relleno efectuado con las salmueras saturadas. Así lo indicaron las nivelaciones realizadas de forma sistemática en el conjunto del barrio por el Institut Cartogràfic de Catalunya (ICC) (1), que indican zonas con valores medios de subsidencia de 2,8 cm/año durante el periodo 1999-2006 y valores que alcanzan los 5,7 cm durante el año 2008. Las medidas de deformación a vista de satélite realizadas durante el periodo 1992-2004 por la Agencia Espacial Europea corroboraban los datos de las nivelaciones superficiales realizadas por el ICC en dicho periodo(4) (5) ${ }^{2}$.

En la Figura 2 se ha reproducido el plano confeccionado por el ICC en el que se muestra la ubicación de los puntos de nivelación en superficie, del taquímetro, de los prismas y de los extensómetros dispuestos en el barrio a lo largo del periodo comprendido entre 1999 y 2008. La información obtenida por esta extensa red de control de movimientos permitía trazar curvas de isodescenso superficial cada $8 \mathrm{~m}$ y verificar movimientos en profundidad del terreno de origen kárstico en la zona crítica coincidente o próxima a la ya citada Gran Cavidad, lugar donde se presentaban los mayores valores absolutos de descenso, que alcanzan el valor de $136 \mathrm{~mm}$. Las zonas de mayor diferencia relativa de descensos se observan donde las distancias entre curvas sucesivas son mínimas. La parte rayada corresponde a la proyección en vertical de la zona con presencia de galerías subterráneas excavadas.

\section{LA INCIDENCIA SOBRE LOS EDIFICIOS DE LOS MOVIMIENTOS DEL TERRENO}

La aparición de lesiones en los edificios es manifestada por los vecinos hacia el año 1995, y su persistencia aconsejó al Ayuntamiento de Sallent realizar en el año 2000 un primer informe sobre las afectaciones visibles presentes, en el que se incluye un plano parcelario del barrio de La Estación con indicación del nivel de daños en cada uno de ellos (6). A partir de este informe y de los datos sobre la subsidencia del terreno, obtenidos con instrumentación taquimétrica y extensométrica, en el año 2001 se procedió al desalojo de los edificios con lesiones más graves, el cual afectó a 11 familias. Sin embargo, fue el estudio completo y exhaustivo del fenómeno realizado por el ICC (1), finalizado en 2002, el que puso de manifiesto en sus conclusiones, de forma clara y concisa, la más que probable persistencia del movimiento de subsidencia de los terrenos ubicados sobre la antigua zona minera en los años futuros, dotando a la Administración de los argumentos científicos necesarios para la toma de decisiones sobre el futuro del barrio y sobre la conveniencia de adoptar medidas de prevención a corto y medio plazo.

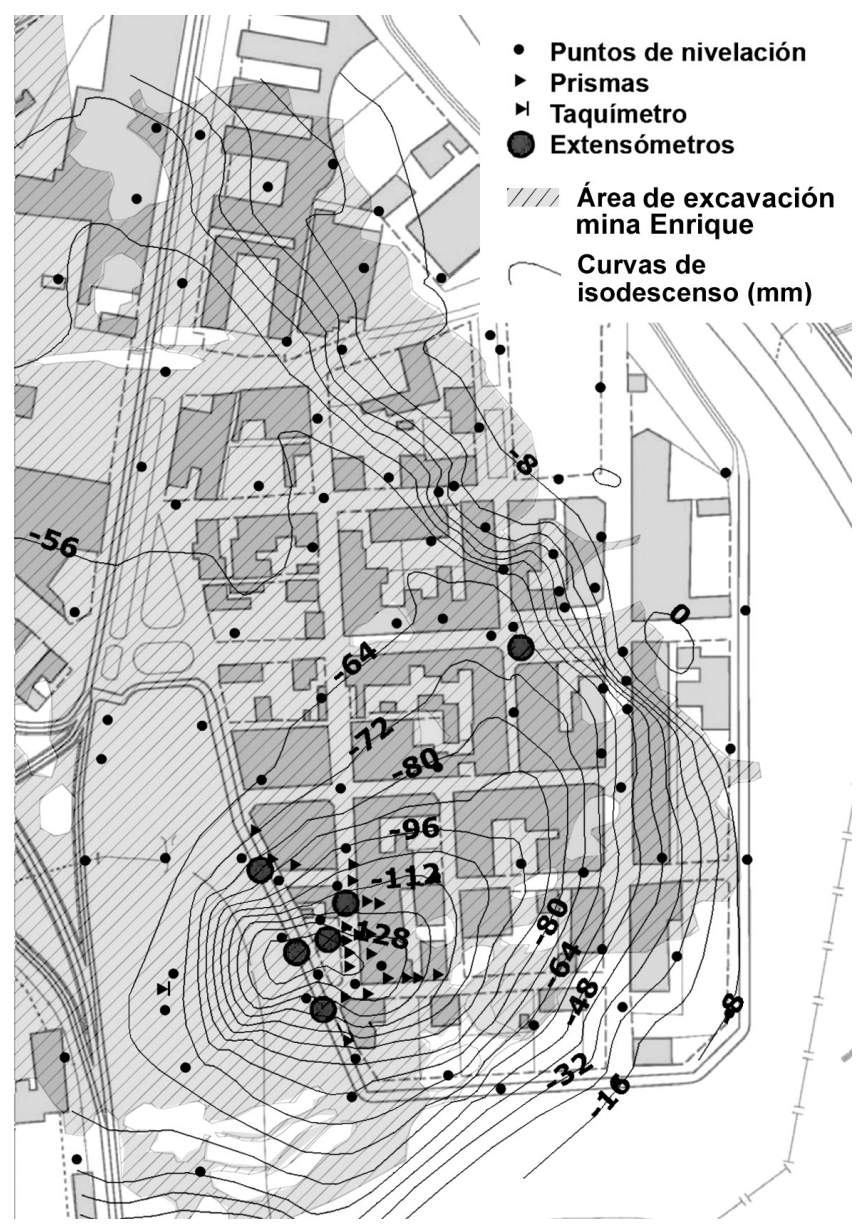

Figura 2. Localización de la instrumentación, curvas de isodescenso y valores absolutos de los descensos en el periodo 1999-2004.

\footnotetext{
${ }^{2}$ Las velocidades medias de subsidencia en el periodo 1948-88 aparecen en un informe realizado por Potasas del Llobregat S.A. citado en el estudio del ICC, mientras que los valores indicados para el periodo 1965-75 provienen de la comunicación presentada por J. Moya et al. en el Congreso Mundial de la Minería de 1979 según se cita en el mismo estudio. Las medidas obtenidas a vista de satélite se exponen y comentan en el artículo de O. Mora et al. en la Revista Catalana de Geografía. El procedimiento aplicado, utilizado las modernas técnicas DinSAR es detalladamente expuesto y comentado en el artículo de E. Sillerico, M. Marchamalo et al. en la revista Informes de la Construcción.
} 


\section{OBJETIVOS Y METODOLOGIAA DE LA INTERVENCIÓN PREVENTIVA EN LOS EDIFICIOS}

Poco tiempo después del conocimiento de las conclusiones del mencionado estudio, se encargó al Departamento de Construcciones Arquitectónicas I de la UPC la elaboración de informes individuales sobre el estado de cada uno los edificios del barrio, y el establecimiento de las actuaciones cautelares y de prevención concretas a llevar a cabo en el futuro en cada uno de ellos (7), hasta el anunciado traslado de la población a alojamientos alejados del área de subsidencia. A continuación se exponen las fases y contenidos de dichas actuaciones, así como el análisis de la información y los datos obtenidos.

\subsection{Levantamiento gráfico completo de las lesiones en los edificios}

En una fase inicial, se realizó el levantamiento planimétrico de las plantas y secciones de los edificios, con indicación de su distribución funcional y la descripción de sus elementos estructurales, de cerramiento exterior y de la obra gruesa en general. Sobre estos planos se detallaban los daños de cada edificio. Dicha información permitía establecer un diagnóstico de las causas de las lesiones visibles, y detectar con suficiente precisión los edificios afectados por movimientos del terreno.

En relación a las técnicas constructivas aplicadas en los edificios, se constató que un gran número de ellos estaban construidos a base de muros de carga de fábrica de ladrillo de 15 o $30 \mathrm{~cm}$ de espesor perforado o hueco paralelos a fachada y apeados en planta baja por pórticos formados con pilares de ladrillo y jácenas de acero u hormigón. Las paredes medianeras y las cajas de escaleras estaban formadas por fábrica de ladrillo de $15 \mathrm{~cm}$, constituyendo, de hecho, los elementos arriostrantes verticales del sistema. Los forjados eran a base de viguetas de hormigón en las parcelas individuales y cerámicos en los bloques de vivienda colectiva.

Los esquemas de fisuración permitían establecer con nitidez los focos de asentamiento diferencial máximo de la edificación. Dichos esquemas se caracterizaban en muchos edificios por la presencia de grietas inclinadas con origen en esquinas de puertas o ventanas y fuerte incidencia en su manifestación de las tensiones de corte. Como puede observarse en la Figura 3 los esquemas abarcaban a veces diversos edificios de una misma calle. En algunas zonas se correspondían con roturas de pavimentos urbanos y muros de separación. En general, los esquemas concordaban con los clásicos modelos de distribución de isostáticas de compresión en elementos verticales a base de materiales elásticos y rígidos (8). De todas formas, en un apreciable número de edificios, el movimiento de subsidencia generó basculamientos globales observables en el desplome de sus fachadas, los cuales en algunos casos se presentaban de forma simultánea a los esquemas de fisuración habituales y en otros aparecían prácticamente como única afectación visible.

\subsection{Plan de seguimiento y control de las condiciones de seguridad de los edificios}

Los datos aportados por el levantamiento de lesiones permitieron disponer de conocimiento preciso de los edificios afectados por asentamientos diferenciales del terreno, priorizar intervenciones cautelares en los edificios en situaciones de riesgo no asumibles por esta causa, y disponer medidas de control de la evolución de los daños en todos los edificios afectados por los movimientos del terreno que permitiesen adoptar actuaciones preventivas en función de los progresivos datos obtenidos. El plan se completaba con la programación de visitas periódicas de inspección a los edificios con el fin de detectar la aparición de nuevas lesiones.

Evidentemente, mientras se elaboraba el informe, el ICC proseguía la toma de datos en el conjunto del barrio sobre la velocidad de subsidencia tanto en superficie como en profundidad. Ello permitió, al combinar dichos datos con los de la inspección visual y la diagnosis de las lesiones, detectar inicialmente el grave estado de algunos edificios y la conveniencia de su desalojo a corto plazo. Esta medida afectó concretamente a dos de los tres bloques lineales, que contenían un total de 122 viviendas, y a un pequeño edificio unifamiliar situado al frente de uno de los bloques. En dichos edificios se constataron cuadros fisurativos generalizados intensos indicando distorsiones angulares ${ }^{3}$ (9) superiores a 1/170 y desplomes que alcanzaban en alguna zona el valor de 1/97 de la altura del edificio. Se aconsejó, también, el apuntalamiento de algunos elementos en edificios con zonas o elementos especialmente dañados.

El establecimiento de estas primeras medidas se avanzó a la finalización de la propuesta del plan de control y seguimiento, que se puso inmediatamente en acción una vez terminado. Dicho plan, esencialmente, concretaba los edificios objeto de seguimiento de la dinámica de las fisuras mediante deformómetros y los plazos de las campañas de medida y de las visitas de reconocimiento, lo cual permitía disponer de los datos con suficiente antelación y poderlos contrastar con los valores de subsidencia suministrados por el ICC.

Para el control de la dinámica de las fisuras originadas por el movimiento del terreno se eligió el uso de deformómetros modelo 543-25oB de la casa Mitutoyo, previa disposición de puntos fijos de referencia de latón adheridos al soporte, a ambos lados de la fisura a controlar, dotados de un contrapunto cónico adaptable a los extremos del aparato para realizar las lecturas. En su elección se tuvo en cuenta su resolución, que alcanza los 0,001 mm, su precisión de lectura, del orden de 0,003 mm, y la versatilidad de colocación que admitían los puntos fijos de posicionado del aparato, capaces de adherirse a casi todos los materiales de los paramentos o de los mismos muros. Este conjunto de características permitían observar con relativa facilidad la incidencia de los ciclos térmicos estacionales en las medidas realizadas, haciendo factible con ello descubrir con mayor nitidez las variaciones de anchura de las fisuras debidas a las causas analizadas relacionadas con el terreno.

\footnotetext{
3 La distorsión angular viene definida en el CTE como «el asiento diferencial entre dos puntos dividido por la distancia que los separa». Dichos valores suelen utilizarse como referencia para las condiciones de asiento relativo entre los elementos de la cimentación, al incidir de una forma directa sobre los esfuerzos generados en las estructuras por la diferencia de asientos. El concepto de distorsión angular y sus iniciales aplicaciones al comportamiento de los edificios bajo el efecto de los asentamientos diferenciales se deben a los profesores A. Skempton y D. MacDonald.
} 

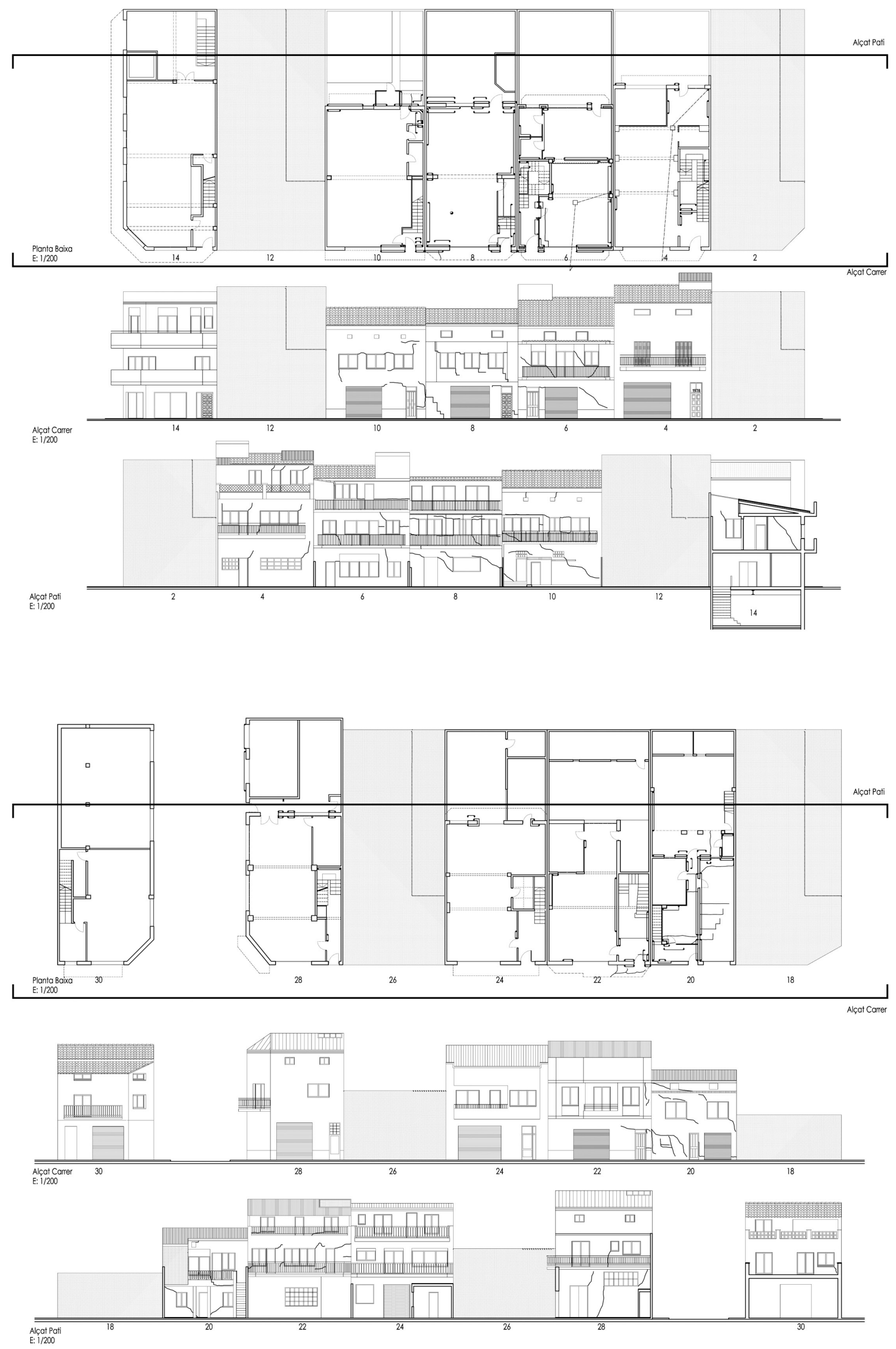

Figura 3. Levantamiento gráfico de los daños en edificios de un tramo de la c. Lleida. 


\subsection{Integración en las previsiones del Plan de Actuación de Emergencias}

A principios del año 2006 la Generalitat de Catalunya aprobó el Plan de Actuación de Emergencias para riesgo de subsidencias en el barrio de L'Estació (Sallent) (10) con el fin de establecer las correspondientes acciones preventivas en las situaciones de riesgo que pudieran presentarse. Dicho Plan, relacionaba y concretaba las actuaciones de alerta diferenciadas en función del alcance de valores límite de velocidad de subsidencia medidos por las redes de instrumentación distribuidas por el barrio. Por lo que se refiere a los puntos de medición mediante deformómetros ubicados en las fisuras de los edificios, establecía el incremento de las campañas de medida dentro de las actuaciones preventivas definidas para las diversas situaciones de alerta. De hecho, siguiendo las indicaciones de alerta establecidas en dicho Plan, en el año 2008 se procedió al desalojo de los edificios de la zona sur del barrio más próximos a la ubicación del extensómetro en el que se sobrepasaron los valores máximos de subsidencia previstos y, también, de un grupo limitado de edificios muy lesionados situado más al norte con incrementos ostensibles de distorsión angular.

\section{EL PROCESO DE SEGUIMIENTO Y CONTROL DE LOS EDIFICIOS}

En la Figura 4 se observan los edificios afectados por asentamientos diferenciales, todos ellos instrumentados con deformómetros. Suman 39 edificios sobre un total de 126 visitados, una vez deducidos los 21 edificios que eran simples cobertizos destinados a garaje o almacén y las 49 casas unifamiliares no visitadas por denegación de permiso o ausencia permanente de sus usuarios. Es fácil ver que la mayor parte de los edificios afectados se ubican en la proximidad de la gran cavidad subterránea y en la zona de máxima pendiente de los niveles de subsidencia.

El control de la dinámica de las fisuras se llevó a cabo con la instrumentación anteriormente descrita. En total se efectuaron 104 campañas de lectura a lo largo del periodo comprendido entre los años 2004 al 2011. Su finalización tuvo efecto cuando la práctica totalidad de los edificios controlados se hallaban ya derribados o desalojados, si bien desde finales de 2008 el número de edificios controlados se redujo considerablemente al producirse el desalojo de un importante número de edificios afectados, coincidiendo con la activación de la Alerta 1 del Plan de Actuación de Emergencias del barrio. El número de puntos de medición colocados fue de 81 , dispuestos en fisuras marcadas en 39 edificios (Figura 4). En 49 de las 81 fisuras controladas, las cuales representan un $60 \%$ del total, se manifestó un progresivo aumento de anchura a lo largo de todo el periodo. Los aumentos de anchura no superaron en ningún caso el valor de $5,7 \mathrm{~mm}$. En la Figura $5 \mathrm{se}$ muestra la evolución de la anchura de las fisuras en algunos de los edificios instrumentados con deformómetros.

\section{ANÁLISIS DE LOS EFECTOS DE LOS MOVIMIENTOS DEL TERRENO SOBRE LOS EDIFICIOS}

Para el análisis de los efectos de los movimientos del terreno sobre los edificios es preciso tener presente ante todo la información disponible y la situación relativa de las galerías

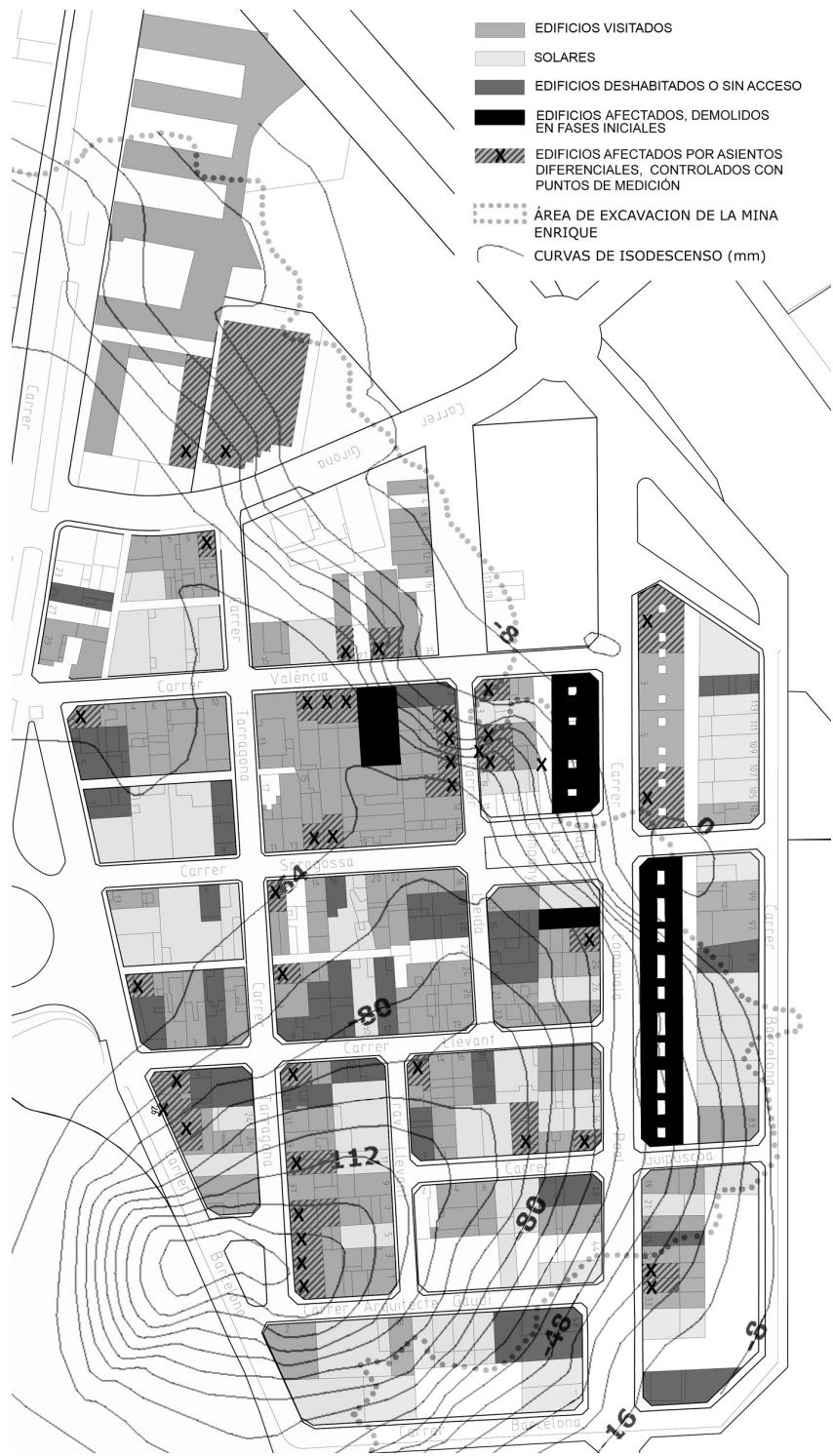

Figura 4. Señalización de los edificios con lesiones de asentamiento e indicación de las características de ocupación de las parcelas.

excavadas en relación a los edificios afectados, puesto que ello condiciona de forma significativa el nivel de deducciones a extraer de los datos obtenidos. En efecto, sobre la localización de las galerías de la denominada mina Enrique, en los estudios geológicos realizados por el Instituto Geográfico de Cataluña se citan profundidades que oscilan entre los 166 y los 280 metros y también la existencia de la anteriormente mencionada Gran Cavidad, que llega a la cota de 279 m, colmatada con materiales desprendidos hasta la cota $216 \mathrm{~m}$, conociéndose con precisión su localización al SO del barrio de la Estación. De todas formas, según se cita en el mismo estudio, no se dispone de ninguna información fehaciente sobre la distribución y las dimensiones de las galerías existentes en el momento del cierre de la mina Enrique, no pudiéndose descartar que la distribución de los vacíos haya variado sensiblemente a lo largo de los años a causa de los efectos destructivos de la disolución de las margas inducida por la presencia de agua. Por otra parte, es preciso tener presente, también, la alta heterogeneidad de los suelos y los procesos de lixiviación de los terrenos salinos situados entre las galerías mineras y los firmes de cimentación de los edificios (1). 

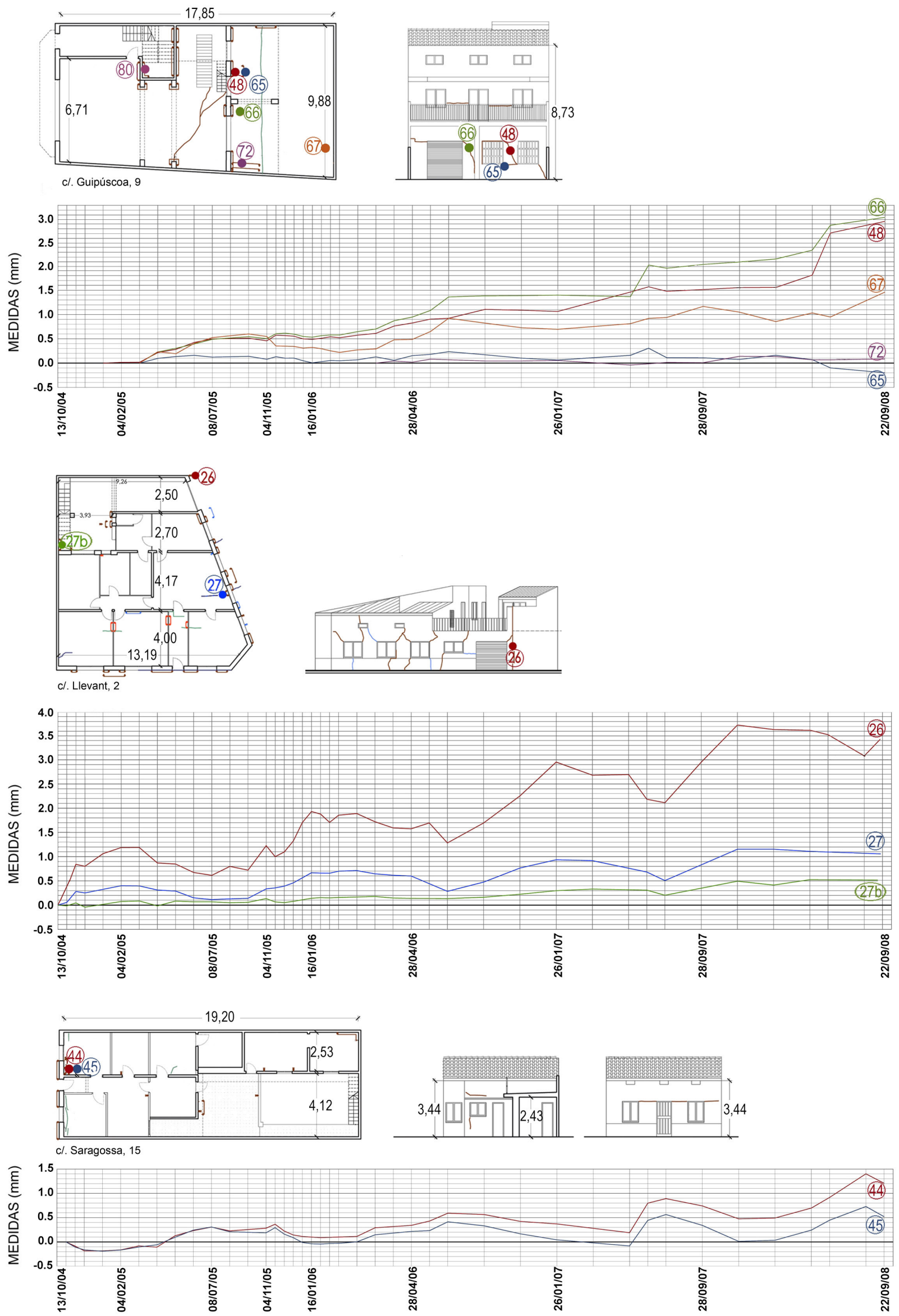

Figura 5. Evolución de anchura de las fisuras instrumentadas en algunos de los edificios afectados. 
Este conjunto de circunstancias y situaciones específicas, en tanto que singularizan el presente caso, impiden adoptar como referentes para el análisis los múltiples estudios existentes sobre los efectos de la excavación y construcción de túneles en situaciones urbanas sobre los edificios. En efecto, en ninguno de los estudios consultados la situación del túnel excavado en relación a la base de los edificios superan los 35 metros medidos en vertical (11) (12) (13) (14), magnitud muy inferior a la de las galerías y cavidades de la mina Enrique. Este hecho, junto a la mencionada heterogeneidad de los terrenos genera, incluso, desigualdades en aquellos que se hallan en firmes de cimentación entre edificios relativamente próximos, lo cual imposibilita la aplicación de los modelos habituales de análisis basados en la conformación de cubetas de asientos en función del tipo de terreno y en la ubicación de los edificios en relación a la excavación del túnel.

Por otra parte, el análisis e interpretación de los cuadros fisurativos de los edificios afectados (Figura 6) muestra que si bien un importante número de los mismos siguieron un movimiento de asiento diferencial en el sentido transversal a las curvas de isodescenso superficial, existían edificios en donde la zona descendida no se correspondía con dicho sentido, aun hallándose ubicados en zonas próximas a la Gran Cavidad subterránea o en áreas en que la subsidencia del terreno generaba mayor pendiente superficial.

Este conjunto de datos y observaciones induce a establecer la presencia de dos escenarios simultáneos en la generación de las afectaciones en los edificios: por una parte, el movimiento general descendente hacia la ubicación de la Gran Cavidad señalado por las curvas superficiales de isodescenso, presente con mayor intensidad en los lugares coincidentes o próximos a la Gran Cavidad y en los de mayor descenso superficial, y por otra, los movimientos más locales, explicables a partir de las heterogeneidades del terreno a profundidades indeterminadas y de la situación del firme de cimentación de los edificios.

Cabe señalar también que los datos obtenidos del seguimiento de las fisuras, si bien ratifican la incidencia del movimiento activo de subsidencia general, muestran un significativo porcentaje de fisuras sin movimiento perceptible a lo largo del periodo en que se hallaron instrumentadas, lo cual da pie a suponer la presencia simultánea de otras causas generadoras de daños directamente asociadas a las deficiencias de su sistema de cimentación o a la acción del agua superficial infiltrada sobre los rellenos de terrenos cohesivos en que se asientan los firmes de las zapatas superficiales de ciertos edificios.

Por lo que se refiere a la forma adoptada por los esquemas de fisuración que presentan los edificios, se observa que la práctica totalidad de los daños aparecidos debidos a asentamiento diferencial corresponden a descensos máximos puntuales o lineales apuntando a los elementos de carga vertical, ya sean pilares, pilastras o muros de ladrillo, y también a situaciones de asentamiento extremo - coincidentes o no con las anteriores - generadoras en los muros de «flexión cóncava hacia abajo», según denominación adoptada en la Tabla 2.2 del Documento Básico «SE-C-Cimientos» del Código Técnico de Edificación (15). No se hallaron, en cambio, cuadros patológicos que indicasen asentamientos centrales generadores de «flexión cóncava hacia arriba» según denominación del mismo Documento. El hecho de que la mayor parte de los edificios dispongan de elementos de carga con separacio-

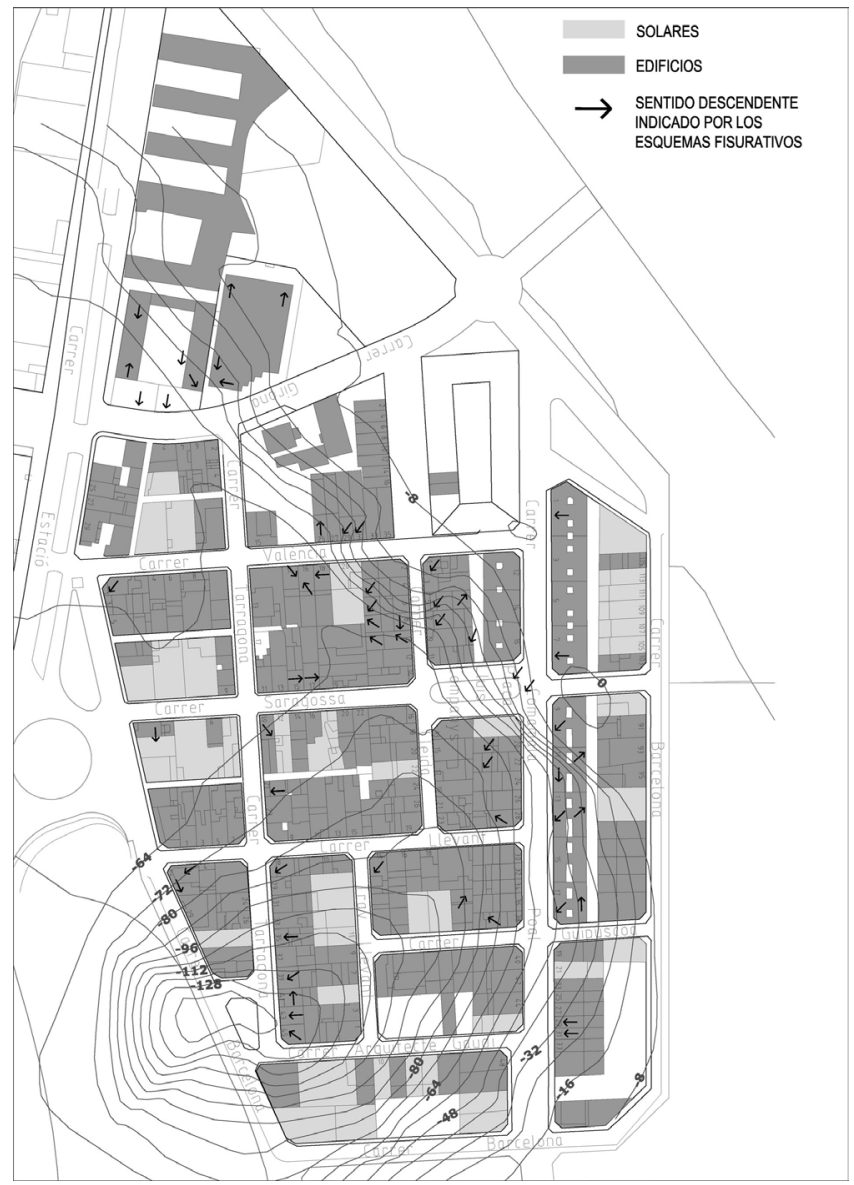

Figura 6. Sentidos descendentes de los movimientos indicados por los esquemas fisurativos de los edificios.

nes máximas del orden de los $6 \mathrm{~m}$ da pie a un principio de comportamiento en el que la incidencia de las cargas sobre la compresibilidad de los terrenos y la tendencia de los muros transversales a seguir el movimiento inducido por los muros de carga predomina sobre los potenciales efectos de los movimientos descendentes de los terrenos exentos de carga situados entre los elementos cargados, descomprimiendo la parte central de los muros, generando con ello «flexiones cóncavas hacia arriba». En todo caso, algunos edificios próximos a la Gran Cavidad, sin presentar esquemas de fisuración demostrativos de este último tipo de flexión, muestran grietas horizontales sintomáticas de las tracciones originadas por pérdidas locales de apoyo, lo cual da pie a suponer la existencia de relaciones más concretas que las consideradas en el Código Técnico entre las características de los edificios y los modelos de movimiento por asientos diferenciales, siendo muy factible que los comportamientos asociados a «flexiones cóncavas hacia arriba» se puedan relacionar más directamente con edificios de mayor longitud que los afectados.

El análisis de los datos sobre la anchura de las fisuras y sus variaciones a lo largo del periodo de tiempo en que fueron instrumentadas debe partir del hecho que no se dispone de información fehaciente del momento de su aparición, y en consecuencia, se desconocen los valores de los asientos de los edificios en dicho momento, lo cual impide el establecimiento de hipótesis sobre los valores críticos de distorsión angular de los elementos de la cimentación relacionables con la formación de fisuras en los mismos. Es por ello que para la interpretación 
práctica de los datos suministrados por los deformómetros se partió del conocimiento de la anchura de las fisuras en el comienzo del periodo de mediciones y de la suposición de un valor de distorsión angular de 1/300 previo al momento de su aparición. Dicho valor, deducido inicialmente por Skempton y MacDonald (9) y ratificado años más tarde por Bjerrum (16) y otros autores es el que suele adoptarse como de referencia cuando - como en el presente caso - no es posible fundamentarse en modelos más afinados ${ }^{4}(17)$. Cabe hacer mención que los valores de distorsión angular que figuran en el Código Técnico de la Edificación (15), se refieren sólo a los valores límite en servicio a especificar en el proyecto, mientras que los de Skempton, MacDonald y Bjerrum incluyen valores límite de riesgo y de fisuración, siendo este último el que se aplicó.

A partir de este valor y del conocimiento de las variaciones de anchura halladas, unido al de las anchuras medidas en el momento de colocar la instrumentación, permitió deducir con razonable aproximación las distorsiones angulares acaecidas en los edificios. En el cuadro de la Tabla 1 se han calculado dichas distorsiones en la fecha de la última campaña efectuada de lectura de los deformómetros, considerando, como se ha expuesto, el valor de 1/300 para la distorsión angular previa a la aparición de las fisuras.
Siguiendo un procedimiento similar, otro conjunto de valores de distorsión angular se halló a partir de la medida directa de la anchura de fisuras durante las últimas visitas de control efectuadas a los inmuebles, adicionando igualmente la distorsión previa a su formación de 1/300, anteriormente indicado. Dichos valores se incluyen también en el cuadro de la Tabla 1.

Se constata que en todos los casos las distorsiones angulares obtenidas fueron inferiores a 1/150, es decir, se mantuvieron inferiores a los valores considerados límite para la seguridad de los edificios con tipologías estructurales de muros de carga por las mismas fuentes anteriormente citadas. De todas formas, la aparición de nuevas fisuras unida al aumento de anchura de las existentes y su previsible crecimiento futuro, aconsejó la adopción de medidas cautelares adicionales en forma de apuntalamientos parciales en las zonas críticas de algunos edificios, aunque los daños aparecidos manifestaran rangos de severidad calificables de $\operatorname{moderados}^{5}(18)$.

Cabe observar, por otra parte, que el orden de magnitud de la anchura máxima alcanzada por las fisuras en su proyección vertical (Tabla 1), es muy inferior al $50 \%$ del de los descensos superficiales del terreno medidos por la instrumentación taquimétrica, los cuales llegan a alcanzar los 128 mm según

Tabla 1. Distorsiones angulares de los edificios del barrio más afectados por la subsidencia durante el periodo de seguimiento y control (2004-2008).

\begin{tabular}{|c|c|c|c|c|c|c|}
\hline Localización & $\begin{array}{l}\text { Ancho inicial } \\
\text { fisura }(\mathrm{mm}) \\
\quad(2004)\end{array}$ & $\begin{array}{c}\text { Incremento } \\
\text { medido en } \\
\text { deformómetro } \\
(\mathbf{m m}) \\
(2004-2008)\end{array}$ & $\begin{array}{c}\text { Ancho máximo } \\
\text { medición } \\
\text { directa }(\mathbf{m m}) \\
(\mathbf{2 0 0 8 )}\end{array}$ & $\begin{array}{l}\text { Inclinación } \\
\text { respecto a } \\
\text { horizontal }\end{array}$ & $\begin{array}{c}\text { Luz } \\
\text { estructural } \\
(\mathbf{m m})\end{array}$ & $\begin{array}{l}\text { Distorsión } \\
\text { angular }\end{array}$ \\
\hline Tarragona, 45 & 2 & 5,95 & - & $45^{\circ}$ & 4180 & $1 / 214$ \\
\hline Tarragona, 45 & - & - & 10 & $45^{\circ}$ & 4180 & $1 / 228$ \\
\hline Guipúscoa, 9 & 3 & 3,10 & - & $45^{\circ}$ & 3100 & $1 / 212$ \\
\hline Guipúscoa, 9 & - & - & 10 & $45^{\circ}$ & 3100 & $1 / 178$ \\
\hline Lleida, 6 & 3 & 1,57 & - & $45^{\circ}$ & 2560 & $1 / 218$ \\
\hline Tarragona, 41 & 4 & 2,13 & - & $45^{\circ}$ & 3100 & $1 / 210$ \\
\hline Tarragona, 41 & - & - & 10 & $45^{\circ}$ & 3100 & $1 / 178$ \\
\hline Tarragona, 43 & & - & 4 & $45^{\circ}$ & 6170 & $1 / 264$ \\
\hline Lleida, 5 & 1 & 2,03 & - & $45^{\circ}$ & 5160 & $1 / 267$ \\
\hline Lleida, 8 & - & - & 5 & $45^{\circ}$ & 5660 & $1 / 252$ \\
\hline Llevant, 2 & - & - & 5 & $60^{\circ}$ & 4000 & $1 / 253$ \\
\hline Saragossa, 15 & 1 & 1,40 & - & $30^{\circ}$ & 2770 & $1 / 245$ \\
\hline Comamala. Poal 28 & - & - & 8 & $30^{\circ}$ & 6400 & $1 / 226$ \\
\hline IES Llobregat & 4 & 1,08 & - & $45^{\circ}$ & 5000 & $1 / 247$ \\
\hline IES Llobregat & 7 & 1,14 & - & $45^{\circ}$ & 5000 & $1 / 223$ \\
\hline
\end{tabular}

\footnotetext{
${ }_{4}$ El valor de 1/30o fue establecido por Skempton y MacDonald y ratificado años más tarde por Bjerrum como valor límite de distorsión angular vertical entre dos puntos de la cimentación del edificio en el momento de aparición de la fisura. Se trata de referencias que, si bien aproximadas, suelen aún adoptarse cuando la heterogeneidad del terreno, el desconocimiento o las insuficiencias de información de los procesos de perforación y de localización de los vacíos subterráneos desaconseja adoptar los valores deducidos de modelos más afinados, adoptados generalmente para la predicción de los movimientos en el subsuelo y sus efectos en la edificación superficial en casos de excavación de túneles urbanos, como el desarrollado recientemente por Camós, Molins et al. en su estudio sobre los efectos de la excavación de la Línea 9 del Metro de Barcelona.

5 Para la clasificación de daños visibles, Burland et al. establecen una graduación en 5 niveles en función de la anchura de las fisuras que afectan al edificio, denominando «moderados» los daños del tercer grado, asociados a fisuras comprendidas entre los 5 y los $15 \mathrm{~mm}$ Los daños de dicho nivel y de los siguientes se considera que son originados en la mayor parte de los casos por movimientos del terreno. Los daños del cuarto nivel y quinto nivel se considera que afectan de una forma directa a las condiciones de seguridad del edificio.
} 
puede observarse en la Figura 2. Si bien la anchura de las fisuras en su proyección vertical no tiene necesariamente que corresponderse de una forma exacta con la magnitud de los asientos verticales, es evidente que la diferencia de los valores comparados supera ampliamente los valores adoptados como de referencia para el establecimiento de los límites de asentamiento máximo en función del tipo de terreno y las características de rigidez de los edificios (19). Esta circunstancia ratifica la ya mencionada singularidad que pueden presentar los fenómenos de subsidencia en relación a los casos habituales de daños causados por las acciones mecánicas directas provenientes de los edificios.

Finalmente, otro de los efectos que se pudieron observar en algunos edificios fue la tendencia a experimentar basculaciones globales del conjunto del inmueble o de un sector considerable del mismo. Dichos efectos generaron desplomes en cerramientos verticales de fachadas o patios que llegaron a ser de orden superior a 1/100 de su altura (Tabla 2), lo cual aconsejó adoptar niveles de control rigurosos o medidas cautelares adicionales al superar los valores límites establecidos por algunas fuentes documentales, tales como los citados en el artículo 4.3.3.2 del Documento Básico DB-SE del Código Técnico de la Edificación que se refiere a valores máximos de $1 / 250$ de la altura total del edificio para que su estructura mantenga suficiente rigidez lateral ante cualquier combinación de acciones (20), o las recomendaciones mucho más restrictivas de las Fichas Técnicas de la desaparecida Oficina Consultora de Estructuras del Colegio de Arquitectos de Cataluña, que cita el valor límite de 1/750 de la altura del edificio (21); o las de M. Weaver, que limita a 1/50o el valor máximo aceptable en muros (22).

Tabla 2. Desplomes alcanzados por edificios del barrio.

\begin{tabular}{|l|c|}
\hline \multicolumn{1}{|c|}{ Localización } & Desplome \\
\hline Comamala Poal, 15 & $1 / 97$ \\
\hline Lleida, 6 & $1 / 175$ \\
\hline Lleida, 5 & $1 / 126$ \\
\hline Valencia, 31 & $1 / 110$ \\
\hline Llevant, 2 & $1 / 160$ \\
\hline
\end{tabular}

\section{CONCLUSIONES FINALES}

Las formas concretas del comportamiento experimentado por los edificios ubicados sobre la excavación minera se deben a factores de difícil interpretación global, dada la gran profundidad y el desconocimiento de los recorridos de las galerías, la heterogeneidad de los terrenos y la propia complejidad del proceso de subsidencia acaecido. Todo ello hace inviable el análisis de las consecuencias del fenómeno a partir de modelos de referencia conocidos y contrastados, fundamentados en la relación entre la localización de las galerías y los asientos inducidos por la excavación. Ahora bien, lo que ha sido posible ratificar con los datos obtenidos es que la causa general de los daños en la mayor parte de los edificios deriva de la subsidencia del terreno, y que a esta causa general hay que adicionar la singularidad de las situaciones locales, tanto las derivadas del terreno como de los propios edificios, para explicar de una forma completa los procesos patológicos acaecidos.

En relación a las formas de manifestación de los movimientos de asentamiento diferencial, se constató la tendencia prioritaria de los edificios -en su mayor parte con luces estructurales menores de $6 \mathrm{~m}-$ a experimentar roturas apuntando descensos hacia los elementos de carga, con claro predominio de las lesiones inclinadas por los efectos de las tensiones de corte. Por otra parte, un significativo número de edificios se comportaron como cuerpos rígidos unitarios, basculando globalmente, a pesar de que de sus características estructurales y constructivas resultasen predecibles esquemas de fisuración más acordes con el alto grado de isostatismo proveniente de sus vínculos. Cabe deducir de lo anterior que estos dos modelos de comportamiento frente a los asientos diferenciales son los dominantes en los tipos de edificios residenciales de hasta 4 plantas de altura con estructura de muros de carga.

Por último, se verificó que los descensos medidos en los puntos de las redes de nivelación superficial instaladas en la zona, fueron de un orden muy superior a los que supuestamente corresponderían a las relaciones habitualmente consideradas entre asientos totales homogéneos y asientos diferenciales deducibles a partir de los valores de las componentes verticales de la anchura de las fisuras, lo cual se puede interpretar como un indicio más de la singularidad del fenómeno de subsidencia acaecido o como una experiencia a considerar para la relativización de los niveles de confianza de las susodichas relaciones de común aplicación.

\section{AGRADECIMIENTOS}

Agradecemos al Institut Català del Sol (INCASOL) su colaboración y confianza a lo largo de todas las fases del desarrollo del convenio suscrito con el Departamento de Construcciones Arquitectónicas I de la UPC. Agradecemos, también, a los Ingenieros de la Edificación Sr. Joan Leiva Navarro y Sr. Marc Tous Coll del Laboratorio de Materiales de Construcción de la Escuela Politécnica Superior de Edificación de Barcelona su esfuerzo en el seguimiento y control de los daños. Finalmente, queremos manifestar nuestro reconocimiento a los técnicos del Ayuntamiento de Sallent y a los miembros de la Asociación de Vecinos del Barrio de la Estación por la ayuda y las facilidades recibidas en el desempeño de nuestra labor.

\section{REFERENCIAS}

(1) Institut Cartogràfic de Catalunya. (2002). Estudi del procés d'esfondrament del terreny als barris de l'Estació i La Rampinya. ICC/AR-065-02 Generalitat de Catalunya.

(2) Batlle \& Mascareñas Geoprojectes. (1995). Diagnosi geotècnica inicial referent a l'aparició de lesions generalitzades en alguns edificis del sector de l'Estació a Sallent, El Bages.

(3) Mata, R., Puiguriguer, M. (2008) Informe geològic, geotècnica i de riscos geològics. Pla d'ordenació urbanística municipal. Departament d'Enginyeria Minera i Recursos Naturals de la UPC - Axial geologia i medi ambient S.L. 
(4) Mora, O., Palà, V., Arbiol, R., Adell, A., Torre, M. (2007). Medidas de deformación del terreno a vista de satélite. Revista Catalana de Geografía, 4 época, 12(31).

(5) Sillerico, E., Marchamalo, M., Rejas, J. G., Martínez, R. (2010). La técnica DInSAR: bases y aplicación a la medición de subsidencias del terreno en construcción. Informes de la Construcción, 62(519): 47-53, doi: http://dx.doi.org/10.3989/ ic.09.063.

(6) Font, M., Ajuntament de Sallent. (2000). Informe sobre el barri de l'Estació de Sallent. Sallent: Ajuntament de Sallent.

(7) Departamento de Construcciones Arquitectónicas I - Universidad Politécnica de Cataluña. (2004). Diagnóstico de los edificios del barrio de La Estación de Sallent. Barcelona: Convenio de Colaboración UPC-INCASOL.

(8) Uriel-Ortiz, A. (1983). Patología de las cimentaciones. Informes de la Construcción, 35(350): 5-35, doi: http://dx.doi. org/10.3989/ic.1983.v35.i350.2019.

(9) Skempton, A.W., Macdonald, D.H. (1956). The allowable settlements of buildings. En ICE Proceedings: Engineering Divisions, 5(6): 727-768, doi: http://dx.doi.org/10.1680/ipeds.1956.12202.

(10) Pla de Protecció Civil de Catalunya (PROCICAT). (2005). Pla d'actuació d'emergències per risc de subsidències en el barri de l'Estació (Sallent).

(11) Attewell, P.B., Yeates, J., Selby, R. (1986). Soil Movements Induced by Tunnelling and their Effects on Pipelines and Structures. New York: Chapman and Hall.

(12) AA.VV. (1984). Ground Movements and Structures: Proceedings of the $3^{\text {rd }}$ International Conference held at The University of Wales Institute of Science and Technology, July 1984. London. Ed. James D. Geddes - The University of Wales Institute of Science and Technology.

(13) Burland, J.B. (2008). The assessment of the risk of damage to buildings due to tunneling and excavations. En Jornada Técnica PaymaCotas - Movimientos de edificios inducidos por excavaciones: criterios de daño y gestión del riesgo (pp. 3-26).

(14) Giardina, G., Marini, A., Hendriks, M., Rots, J. G., Rizzardini, F., Giuriani, E. (2012). Experimiental analysis of a masonry façade subject to tunnelling-induced settlement. Engineering Structures, 45: 421-434, doi: http://dx.doi.org/10.1016/j. engstruct.2012.06.042.

(15) CTE. (2006). Real Decreto 314/20o6. Documento Básico SE-C Cimientos. Código Técnico de la Edificación.

(16) Bjerrum, L. (1963). Discussion on Proceedings of European Conference on Soil Mechanics and Foundation Engineering vol III. Norwegian Geotechnical Institute Publ. (98): 1-3.

(17) Camós, C., Arnau, O., Molins, C., Alegre, V. (2011). Estudio de las cubetas de asientos producidas por la tunelación y simulación de la respuesta estructural de edificios afectados. En V Congreso ACHE. Barcelona.

(18) Burland, J.B. (1977). Behavior of foundations and structures. State of the Art Report. En Proc. 9th Conf. On Soil Mechanics anf Foundations Engineering, II, (pp. 495-546). Tokyo: Japanese Geotechnical Soc.

(19) Mañá, F. (1978). Patología de las cimentaciones. Barcelona: ed. Blume.

(20) CTE. (2006). Real Decreto 314/2006. Documento Básico SE-Seguridad Estructural. Código Técnico de la Edificación.

(21) OCE - Colegio de Arquitectos de Cataluña (1974). Asientos admisibles en los edificios, Ficha núm. 30 (Fichas Técnicas de Construcción). Barcelona: Oficina Consultora de Estructuras (OCE) del Colegio de Arquitectos de Cataluña.

(22) Weaver, M. (1997). Conserving buildings, a manual of techniques and materials. Canada: J. Wiley \& Sons. 